Türkiye Jeoloji Bülteni
Geological Bulletin of Turkey
$62(2019) 1-16$
doi: $10.25288 /$ tjb.496325

\title{
Palu (Elazığ) Civarında Yüzeylenen Bitlis Masifi Üst Triyas Karbonatlarının Foraminifer Biyostratigrafisi, Mikrofasiyes Analizleri ve Çökelim Ortamları
}

Foraminiferal Biostratigraphy, Microfacies Analysis and Depositional Environments of Upper Triassic Carbonates of Bitlis Massif, Palu (Elazı̆̆)

\author{
Ayşe Atakul Özdemir \\ Van Yüzüncü Yıl Üniversitesi, Jeofizik Mühendisliği Bölümü, Van
}

Geliş/Received : 21.10.2018 • Düzeltilmiş Metin Geliş̧/Revised Manuscript Received : 22.11.2018 • Kabul/Accepted : 26.11.2018 • Bask1/Printed : 01.01 .2019 Araştırma Makalesi/Research Article Türkije Jeol. Bül. / Geol. Bull. Turkey

Öz: Bu çalışmada, Elazı̆̆ il sınırları içinde Çakmakkaya köyü (Palu, Elazı̆̆) civarında yüzeylenen Bitlis masifi örtü birimleri içerisinde yeralan Üst Triyas karbonatlı kayaçların mikrofasiyes özelliklerinin ve foraminifer içeriklerinin ortaya konulması amaçlanmıştır. Ölçülen kesit boyunca kireçtaşlarından Triyas'ı karakterize eden stratigrafik dağılımları açısından büyük öneme sahip olan foraminifer türleri tayin edilmiştir. İstif içerisinde kaydedilen Triasina hantkeni Geç Triyas için kılavuz fosil olarak kabul edilmektedir. Klavuz fosile örneklerde, Aulotortus sinuosus, Aulotortus friedli, Involutina sp., Galeanella? sp., Duostomina sp., Glomospira sp., Glomospirella sp., Duotaxis birmanica, Siphovalvulina sp., ve Reophax sp. foraminifer topluluğu eşlik etmektedir. Bu türlere dayanılarak geç Noriyen-Resiyen yaşını veren Triasina hantkeni zonu tanımlanmıştır. Foraminifer türlerinin yanı sıra kesit içerisinde megalodontlar, algler, gastropod ve mercan fosilleri de bulunmaktadır. Bölgede yayılım gösteren bu karbonatların çökelim ortamlarını belirleyebilmek için gerçekleştirilen mikrofasiyes çalışmaları sonucunda; çamurtaşı, vaketaş1istiftaşı ve pelletli biyoklastlı istiftaşı-tanetaşı olmak üzere 3 mikrofasiyes tipi belirlenmiştir. Mikrofasiyes tipleri, çökelimin lagün ve sığ denizel ortamda gerçekleştiğini işaret etmektedir.

Anahtar kelimeler: Bitlis masifi, foraminifer, geç Triyas, mikrofasiyes, Palu

\begin{abstract}
In this study, it is aimed to reveal the foraminiferal content and microfacies properties of the the Upper Triassic carbonates, cover units of the Bitlis massif exposed in the vicinity of the Çakmakkaya village (Palu, Elazığ). Stratigraphically significant foraminiferal species characterizing Triassic have been recorded throughout the measured section. Triasina hantkeni, the most common taxa documented in the studied sequence, is considered as a zonal marker for Late Triassic. Besides the zonal marker species, the section includes Aulotortus sinuosus, Aulotortus friedli, Involutina sp., Galeanella? sp., Duostomina sp., Glomospira sp., Glomospirella sp., Duotaxis birmanica, Siphovalvulina sp., and Reophax sp. foraminiferal assemblages. On the basis of the determined foraminiferal taxa, Triasina hantkeni zone is defined within the studied succession and a late Norian-Rhaetian age is attributed. In addition to foraminiferal assemblages, megalodontids, algae, gastrapods and corals are also present within the limestone units. Three facies types, mudstone, wackestone-packstone, pelloidal bioclastic packstone-grainstone, were described based on microfacies studies to determine the depositional environments of the Upper Triassic carbonates exposed in the region. The described microfacies types suggest that the depositional environment was a lagoon and shallow marine platform environments.
\end{abstract}

Keywords: Bitlis Massif, foraminifera, late Triassic, microfacies, Palu 


\section{GíRIŞ}

Neotetis Okyanusunun Geç Triyas'taki açılımı, Geç Kretase'de meydana gelen dalma-batma mekanizması ile kapanmaya başlaması ve Miyosen'de kıta kıta çarpışması ile yitilmesi, sonrasinda meydana gelen denizel ve gölsel havzalar ile çarpışma sonrası volkanizma Güneydoğu Anadolu Bölgesi ve Doğu Anadolu Bölgesinin jeolojisini şekillendiren en önemli jeolojik olaylardır. Neotetis Okyanusu'nun güney kolunun ilk açılımına yönelik bulgular Toroslarda ve Arap Levhası üzerinde elde edilen veriler yardımıyla ortaya konulmuş ve açılma yaşı Geç Triyas olarak öne sürülmüştür (Robertson vd., 2007, 2016; Varol vd., 2007; Mackintosh ve Robertson, 2012). Bu okyanusun açılımının Bitlis Kenet Kuşağı içindeki varlığını ortaya koyan çalışmalar da bulunmaktadır (Perinçek, 1980; Aktaş ve Robertson, 1984; Yılmaz vd., 1993; Robertson vd., 2007). Perinçek (1980) Geç Triyas yaşlı karbonatlara eşlik eden volkanik kayaçların varlığını göz önünde bulundurarak, Neotetis Okyanusu'nun açılımının Bitlis Masifi içerisindeki izlerinin Elazığ-Palu civarında olabileceğini ileri sürmüştür. Ancak, masifi oluşturan kayaçların Geç Kretase'de metamorfizmaya uğramış olmaları ve çarpışma sonrasında yoğun deformasyonlara maruz kalmaları kayaçların çökelim ortamlarının, dolayısı ile metamorfizma öncesi paleocoğrafyanın tam olarak ortaya konulabilmesini engellemektedir.

$\mathrm{Bu}$ bağlamda, masifte metamorfizmadan az etkilenmiş karbonatlarının bulunması ve bunların fasiyes ve fosil içeriklerinin belirlenmesi masifle ilişkili paleocoğrafik çalışmaların temelini oluşturmaktadır. Bitlis Masifi içerisinde yüzeylenen ve masifin örtü birimleri içerisinde yeralan Geç Triyas yaşlı kireçtaşlarının foraminifer biyostratigrafisinin ve mikrofasiyes özelliklerinin ortaya konulması amaciyla Palu (Elazı̆̆g) güneyinde Çakmakkaya köyü civarında bir stratigrafik kesit (Palu kesiti) ölçülmüştür (Şekil 1). Örneklenen istif genel olarak megalodontlu sı ̆ denizel karbonatlardan oluşmaktadır (Şekil 1). Bitlis Masifinin Üst Triyas foraminifer biyostratigrafisi ilk kez bu çalışma ile ortaya konulmuştur.

\section{JEOLOJI}

Bitlis Kenet Kuşağı geç Mesozoyik-erken Senozoyik aralığında birbiri üzerine yerleşmiş farklı tektonik birimlerden oluşmaktadır (Hall, 1976; Aktaş ve Robertson, 1990; Yılmaz vd., 1993; Robertson, 1998; Okay vd., 2010). Kenet Kuşağını oluşturan birimlerden Arap platformuna ait sedimanter birimleri Bitlis Masifi olarak isimlendirilen metamorfik kayaçlar, Geç Kretase yaşlı ofiyolitik melanj ve Eosen yaşlı filişler üzerlemektedir. Bitlis Masifi, amfibolit, paragnays/flist, eklojit, migmatit seviyeleri ve bu kayaç gruplarını kesen metavolkanitler, granitik ve hololökokratik granitik kayaçlardan oluşan Pre-Kambriyen temel ile bunları geçişli olarak üzerleyen Paleozoyik-Mesozoyik yaşlı karbonatlardan oluşmaktadır (Çağlayan vd., 1984; Göncüoğlu ve Turhan, 1984; Okay vd., 1985; Şengün, 1993).

Çalışma alanı ve yakın çevresinde Paleozoyik-Mesozoyik yaşlı Bitlis Masifi'ne ait glokofan şist, kloritşist, kuvars şist ve bu çalışmanın konusunu oluşturan Üst Triyas sı̆̆ denizel rekristalize karbonatlar temel kayaçlar olarak yüzeylenmektedir (Şekil 2). Kretase yaşlı Guleman ofiyolitik melanj1, dünit, piroksenit, harzburjit, diyabaz, gabro ve bazalt bloklarından oluşmaktadır (Perinçek, 1980). Çalışılan alanda yüzeylenen Orta Eosen yaşlı Maden kompleksine ait birimler diyabaz, bazalt, andezit benzeri volkanik kayaçlar ve yer yer bunlarla ardalanmalı olarak gözlenen çamurtaşı, kiltaşı, kumtaşı ve kireçtaşı ile karakterize edilir. Geç Eosen-erken Miyosen yaşlı türbiditik karakterde kırıntılı kayaçlarla temsil edilen ve kumtaşı, silttaşı, kiltaşı ve marn ardalanmasından oluşan Çüngüş formasyonu ise çalışma alanı ve çevresine yüzeylenen en genç birimdir (Perinçek, 1980, 1990). 

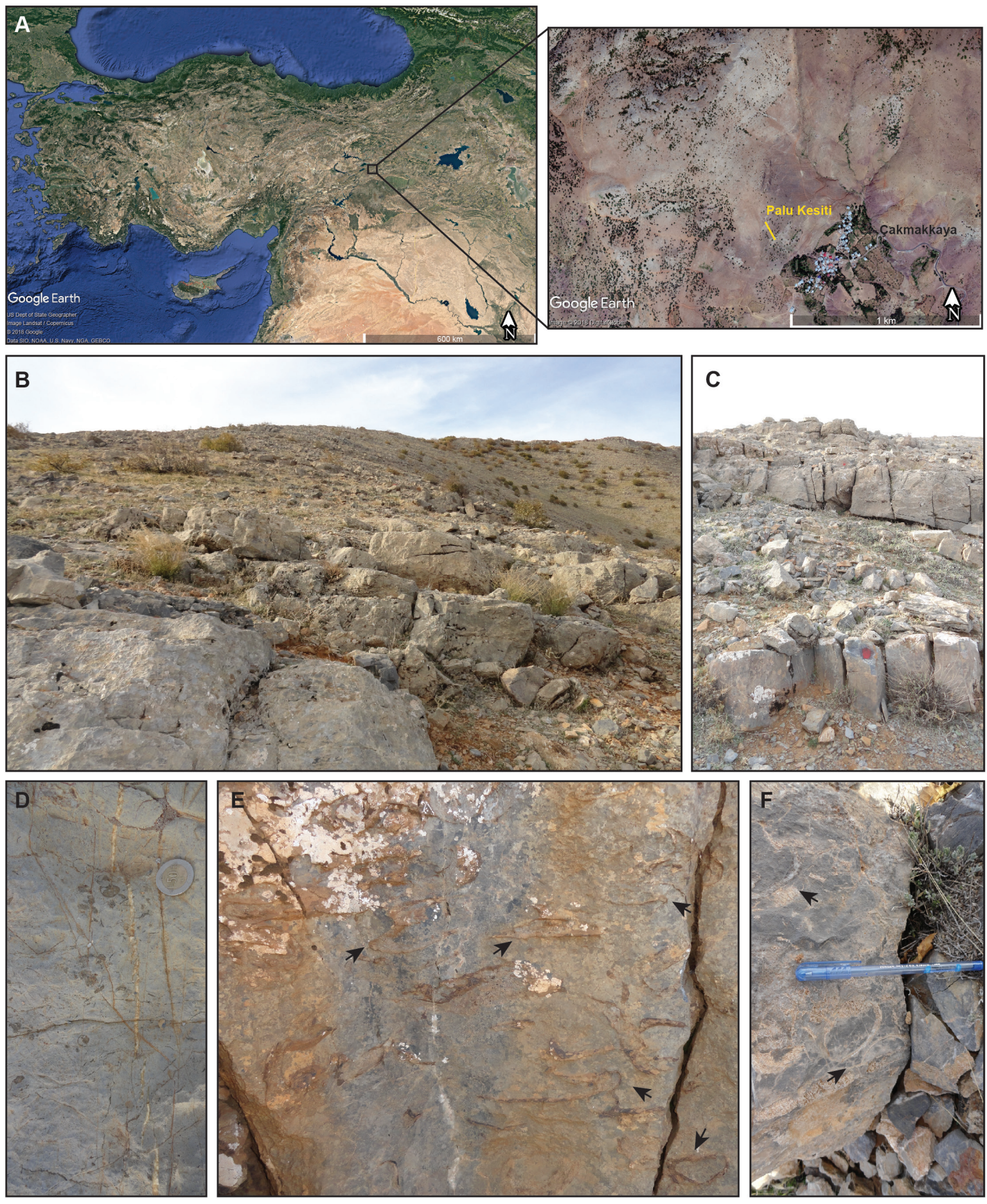

Şekil 1. Çalışma alanının yer bulduru haritası (a), ölçülü stratigrafik kesitin arazi görünümleri (b,c), örneklenen kireçtaşlarının ve içerisinde gözlenen megalodontların yakından görünümü (d,e,f).

Figure 1. Location map of the study area (a), field view of the measured stratigraphic section (b,c), close-up view of the limestones and megalodonts observed within these limestones $(d, e, f)$. 


\section{MATERYAL VE METOD}

Bitlis Masifi içerisinde yer alan Üst Triyas karbonatlı kayaçların foraminifer içeriklerinin ve mikrofasiyes özelliklerinin belirlenebilmesi amaciyla Palu (Elazığ) güneyinde Çakmakkaya köyü civarında stratigrafik kesit ölçülmüş ve örneklenmiştir. Çalışma alanı, 1:25000 ölçekli Elazığ K43c $\mathrm{c}_{3}$ ve $\mathrm{K} 43 \mathrm{c}_{4}$ paftalarında yer almaktadır (Şekil 1, Şekil 2). İstif boyunca alınan kireçtaş1 örneklerinden bentik foraminifer topluluğunun belirlenmesi ve fasiyes tanımlamaları için ince kesitler hazırlanmıştır. Kireçtaşlarının adlandırılmasında Dunham (1962) sınıflamasından yararlanılırken mikrofasiyeslerin ve fasiyes zonlarının belirlenmesinde ise Wilson (1975) ve Flügel (2004) modelleri kullanılmıştır.

\section{FORAMINIFER BIYYOSTRATIGRAFISİ}

Çakmakkaya (Palu, Elazığ) civarında yüzeylenen Üst Triyas birimleri sı ̆ denizel karbonatlar ile temsil edilmektedir (Şekil 3). Bu çalışma ile ölçülen kesit boyunca örneklerin içerdiği foraminifer topluluğu ortaya konulmuştur (Şekil 4). Geç Triyas faunası genel olarak involutinid formlarca zengin olup lagenid ve textularid formları da içermektedir. Bu formların yanı sıra birim içerisinde megaladontlar, gastrapodlar, mercanlar ve algler de mevcuttur. Bölgede yayılım gösteren bu sı̆̆ denizel karbonatlarda, geç Noriyen-Resiyen aralığında indeks fosil olarak tanımlanan ve Tetis sisteminin en önemli türlerinden biri olan Triasina hantkeni tespit edilmiştir. Bu indeks fosil ile birlikte Aulotortus

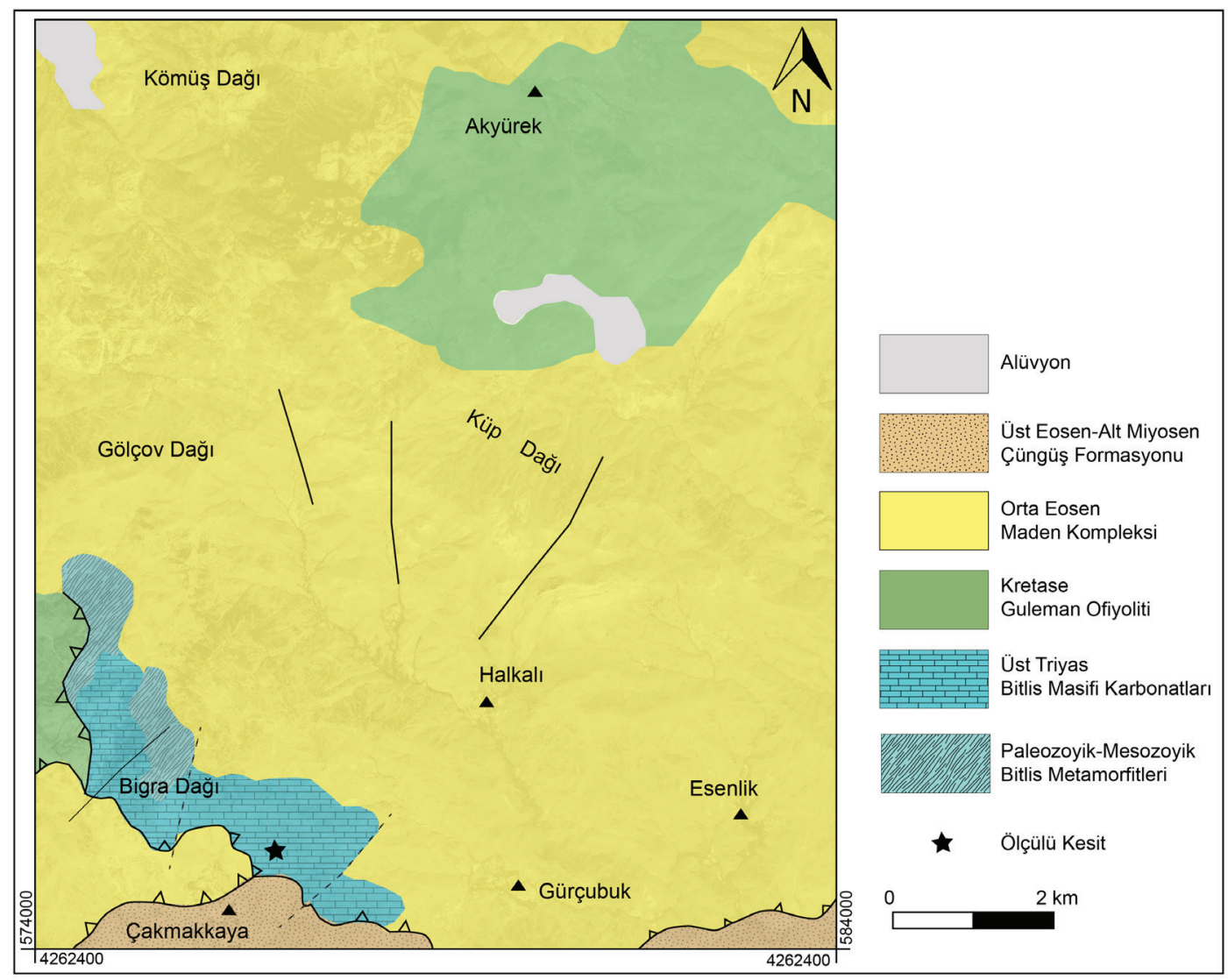

Şekil 2. Çakmakkaya (Palu, Elazı̆̆) civarında yüzeylenen birimlerin jeoloji haritası (MTA, 2011).

Figure 2. Geological map of the units exposed in the study area, around Çakmakkaya (Palu, Elazığ) (MTA, 2011). 
sinuosus, Aulotortus friedli, ve Involutina sp. benzeri involutinid türleri de kesit içerisinde yaygın olarak bulunmaktadır (Şekil 5). Kesit boyunca alınan örneklerden involutinid formlar ile birlikte Galeanella? sp., Duostomina sp., Glomospira sp., Glomospirella sp., Textularia sp., Duotaxis birmanica, Siphovalvulina sp., Reophax sp. türleri de belirlenmiştir (Şekil 5). Tanımlanan foraminifer türlerine dayanılarak çalışılan istifde Triasina hantkeni biyozonu belirlenmiş ve birim yaşı geç Noriyen-Resiyen olarak tespit edilmiştir. Bir çok araştırmacı çalışmalarında Triasina hantkeni biyozununun geç Noriyen-erken Resiyen zaman aralığını kapsadığını belirtmiştir Zaninetti, 1976; Al-Shaibani vd., 1982, 1983, Martini vd., 1997, 2004; Kamoun vd., 1998; Mancinelli vd., 2005). Söz konusu biyozon farklı bölgelerde ve alanlarda yapılan bir çok çalışmada kullanılmakta ve böylece küresel ölçekte korelasyon sağlanmasına olanak sağlamaktadır (Polonya: Gazdzicki, 1974, 1983; Endonezya: Al-Shaibani vd., 1982, 1983; Martini vd., 2004; İtalya: Ciarapica ve Zaninetti, 1984; Mancinelli vd., 2005; Tunus: Kamoun vd., 1994; Türkiye: Okay ve Altıner, 2007; Yümün vd., 2013; Tunaboylu vd., 2014).

\section{MIKKROFASIYYES TIPLERİ VE ORTAMSAL YORUMLAR}

Ölçülen birimlerden alınan örneklerin ince kesitleri detaylı olarak incelenmiş olup petrografik tanımlamalarda Dunham (1962) kullanılırken, mikrofasiyes ve fasiyes zonlarının tanımlamalarında Wilson (1975) ve Flügel (2004) modelleri kullanılmıştır. Detaylı mikrofasiyes çalışmaları sonucunda istif içerisinde çamurtaşı, vaketaş1-istiftaş1, pelletli biyoklastlı istiftaş1tanetaşı olmak üzere üç temel fasiyes tipi belirlenmiştir. Çamurtaşı fasiyesi tamamen mikritten oluşmaktadır ve fosil içermemektedir (Sekil 6 A-B). Bu fasiyes tipi kesit boyunca çok yoğun olarak gözlenmemektedir. Çamurtaş1 fasiyesinin Flügel (2004) tarafindan tanımlanan SMF 23 tipinde olduğu ve düşük enerjili lagün (FZ8) ortamında çökeldiği belirlenmiştir. Vaketaş1istiftaşı fasiyesi genel olarak fosil bakımından fakir olup az miktarda textularid ve lagenid formlar içermekte ve yoğun mikritik matriks içermektedir (Sekil 6 C-D). Fasiyes içerisinde çatlak ve erime boşlukları kalsit dolguludur ve yer yer stilolitleşme gözlenmektedir. Bu nedenlerden dolay1, fasiyes SMF 9-10 standard mikrofasiyes tipine benzerlik göstermekte olup açı platform ortamında (FZ7) çökelmiştir. Pelletli biyoklastlı istiftaşı-tanetaşı mikrofasiyesi istif içerisinde en yaygın fasiyeslerden birini oluşturmaktadır (Sekil 6 E-F). Fasiyes içerisinde pelletlerin yanı sıra bol miktarda involutinid ve textularid formlar ile alg ve mercanlar bulunmakta, bağlayıcı malzeme ise çoğunlukla mikritden oluşmakta ve yer yer sparitik çimento da içermektedir. Ayrıca bazı tanelerin etrafında mikritik zarf bulunmaktadır. İstif boyunca kireçtaşları yer yer kısmen veya tamamen rekristalizasyona uğramış olup içinde bazı kavkı parçaları da gözlenmiştir. İstif içerisinde gözlenen bu fasiyes tip SMF 18 olarak tanımlanmakta ve çökelim ortamı olarak sı̆̆ platform (FZ7) ortam koşullarını yansıtmaktadır. 


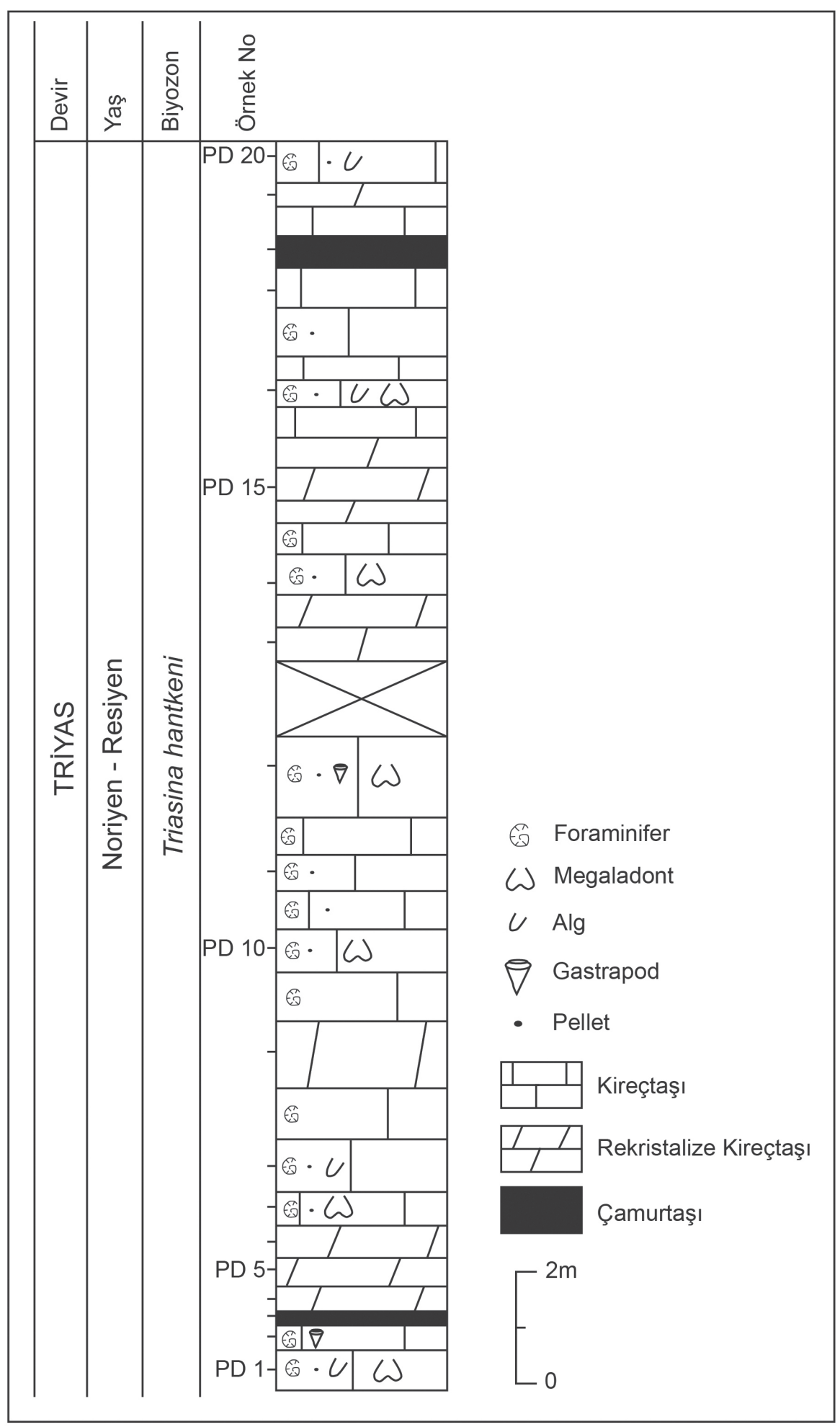

Şekil 3. Palu ölçülü stratigrafik kesiti.

Figure 3. Measured stratigraphical section of Palu. 


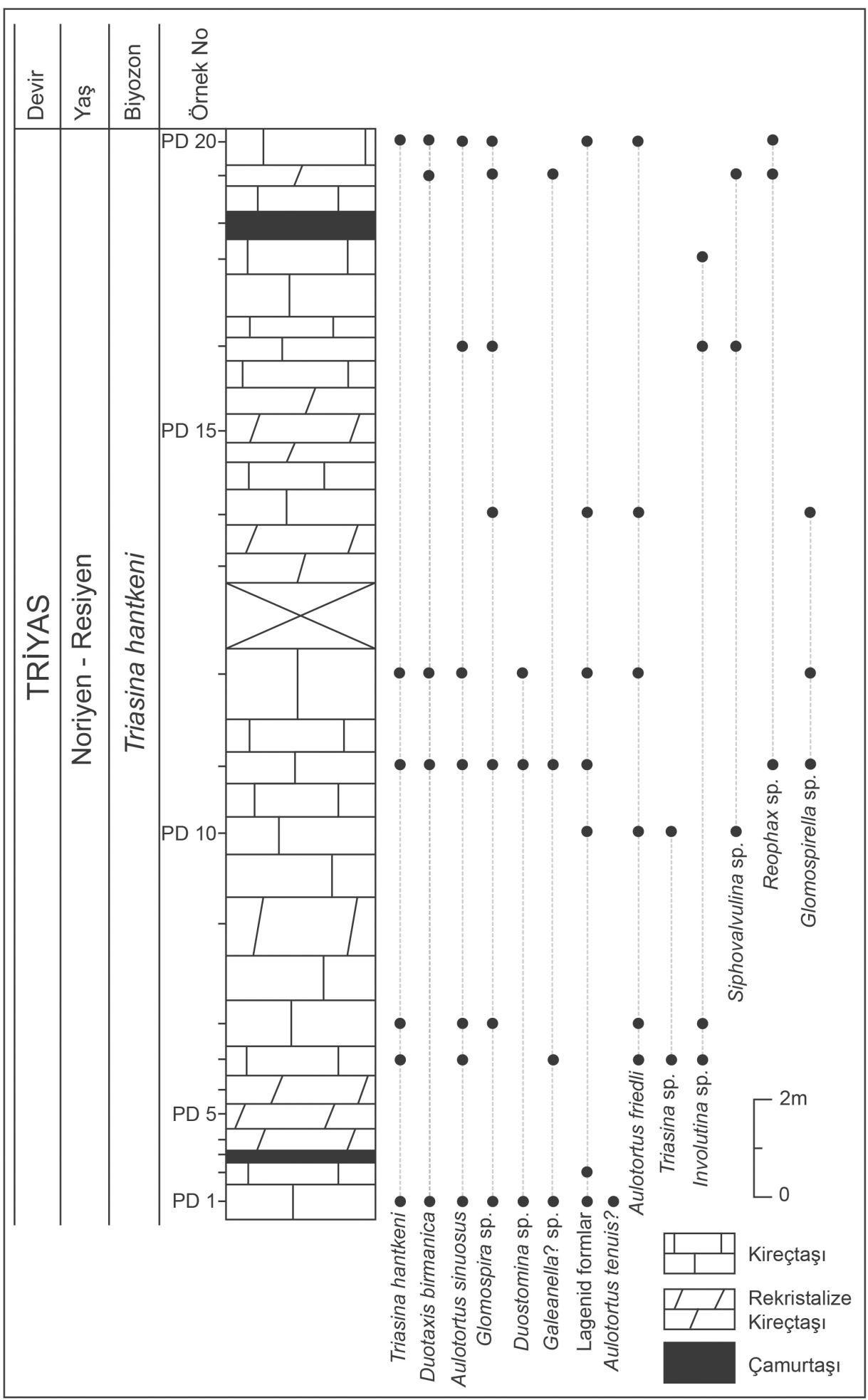

Şekil 4. Palu ölçülü kesiti boyunca tanımlanan foraminiferlerin stratigrafik dağılımları.

Figure 4. Stratigraphic distribution of foraminifera in the Palu measured section. 


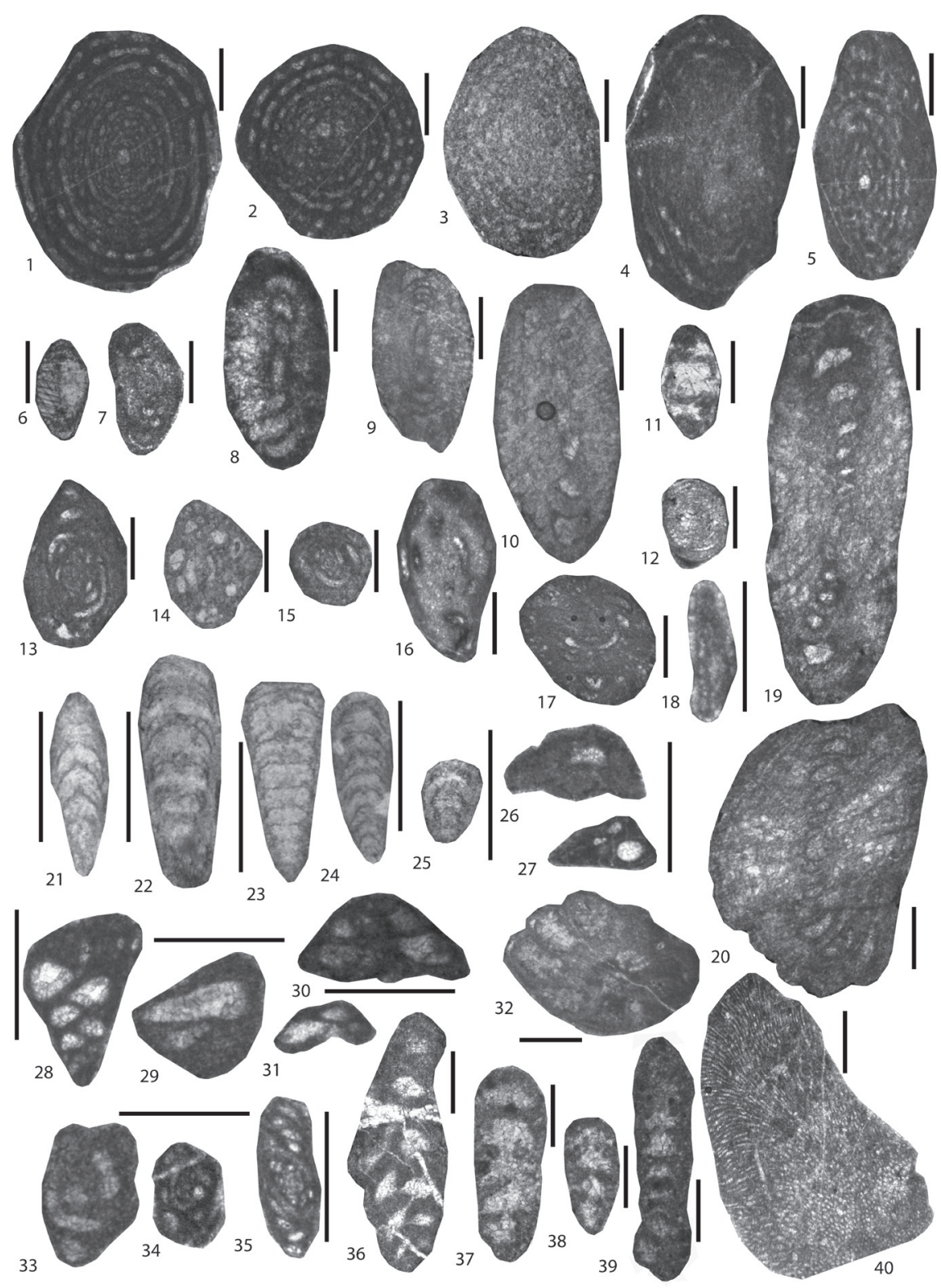

Şekil 5. Üst Triyas karbonatlarından elde edilen foraminifer türleri. 1-4. Triasina hantkeni (1,2: PD 8; 3,4: PD 7), 5. Triasina sp. (PD 7), 6-10. Aulotortus sinuosus (6-8: PD1; 9,10: PD 7), 11-17. Aulotortus friedli (11: PD 12, 14; 12: PD 11; 13, 15-17: PD 7), 18. Aulotortus tenuis? (PD 12), 19-20. Involutina sp. (19: PD 7; 20: PD 8), 21-25. Lagenid formlar (21, 22: PD 23; 23: PD 8; 24, 25: PD 2), 26-27. Galeanella? sp. (PD 11), 28-31. Duotaxis birmanica (28: PD 11; 29: PD 19; 30, 31: PD 20), 32. Duostomina sp. (PD 8), 33-34. Glomospira sp. (33: PD 1; 34: PD 7 ), 35. Glomospirella sp. (PD 11), 36. Siphovalvulina sp. (PD 10), 37-39. Reophax sp. (PD 20), 40. Alg (PD 8).

Figure 5. Foraminiferal species from the Upper Triassic carbonates. 1-4. Triasina hantkeni (1,2: PD 8; 3,4: $P D$ 7), 5. Triasina sp. (PD 7), 6-10. Aulotortus sinuosus (6-8: PD1; 9,10: PD 7), 11-17. Aulotortus friedli (11: PD 12, 14; 12: PD 11; 13, 15-17: PD 7), 18. Aulotortus tenuis? (PD 12), 19-20. Involutina sp. (19: PD 7; 20: PD 8), 21 25. Lagenid formlar (21, 22: $P D$ 23; 23: $P D$ 8; 24, 25: PD 2), 26-27. Galeanella? sp. (PD 11), 28-31. Duotaxis birmanica (28: PD 11; 29: PD 19; 30, 31: PD 20), 32. Duostomina sp. (PD 8), 33-34. Glomospira sp. (33: PD 1; 34: PD 7), 35. Glomospirella sp. (PD 11), 36. Siphovalvulina sp. (PD 10), 37-39. Reophax sp. (PD 20), 40. Algae (PD 8). 

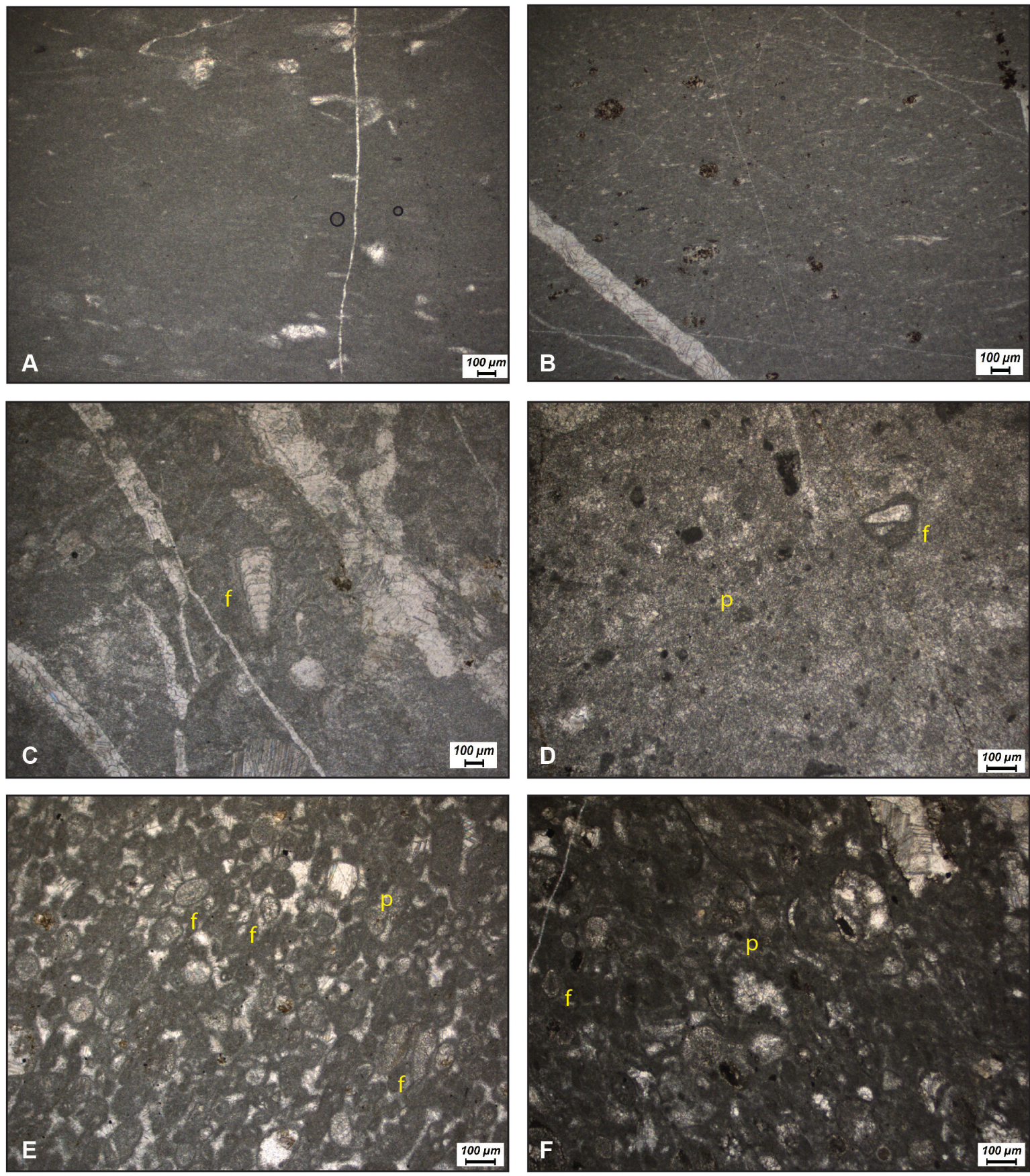

Şekil 6. Üst Triyas karbonatların mikrofasiyes tipleri. A-B. çamurtaşı fasiyesi (A:PD3; B:PD18), C-D. vaketaş1istiftaşı fasiyesi (C:PD2, D:PD22), E-F. pelletli biyoklastlı istiftaş1-tanetaşı fasiyesi (E:PD10, F:PD 12) (f:foraminifer, p:pellet).

Figure 6. Microfacies types of Upper Triassic carbonates. A-B. mudstone facies (A:PD3; B:PD18), C-D. vackestonepackstone facies (C:PD2, D:PD22), E-F. Pelloidal bioclastic packstone-grainstone facies (E:PD10, F:PD 12) (f:foraminifera, p:pellet). 


\section{PALEOCOĞRAFIKK YAKLAŞIM}

Bölgede yay1lım gösteren Üst Triyas karbonatlarına ait mikrofasiyes tipleri, çamurtaşı, vaketaş1-istiftaşı ve pelletli biyoklastlı istiftaşıtanetaşı olarak tanımlanan mikritik fasiyeslerden oluşmaktadır. $\mathrm{Bu}$ fasiyes tipleri yoğun olarak düşük enerjili lagünel ortamlarda çökelmektedir (Şekil 7). Karbonat platformlarında çökelme ortamlarının belirlenmesinde, mikrofasiyes özelliklerinin yanı sıra, foraminiferler de yaygın bir şekilde kullanılmaktadır. Karbonat kayaçları içerisinde gözlenen farklı foraminifer toplulukları spesifik çökelme ortamlarını ve ekolojik faktörleri belirtmektedir (Schäfer ve Senowbari-Daryan, 1981; Márquez vd., 1994; Chablais vd., 2011). Çalışılan Üst Triyas karbonat istifi boyunca tanımlanan involutinid, lagenid ve duostominid formlardan oluşan foraminifer topluluğu, birimin sı ̆g denizel lagün ortamında çökelmiş olduğunu desteklemektedir. Palu (Elazığ) civarında gerçekleştirilen bu çalışmada, bölgede yayılım gösteren karbonatların Türkiye'de farklı bölgelerdeki karbonat istiflerine benzer foraminifer topluluklarına, fasiyes tiplerine ve çökelme ortamlarına sahip olduğu gözlenmiştir (Biga Yarımadası: Beccaletto vd., 2005; Doğu Toroslar: Altıner ve Zaninetti, 1980; Ekmekçi vd., 2006; Yümün vd., 2013; İzmir: Okay ve Altıner, 2007; Karaburun Yarımadası: İşintek, 2002; Tunaboylu vd., 2014). Ayrıca, bu çalışma ile Bitlis masifine ait Üst Triyas birimlerin içerisinde belirlenen foraminifer topluluğu ve fasiyes tipleri dünyada farklı bölgelerde Triyas üzerine gerçekleştirilen çalışmalar ile karşılaştırıldığında, İtalya (Ciarapica ve Zaninetti, 1984; Mancinelli vd., 2005), Batı Karpatlar (Gazdzicki, 1983; Gale vd., 2012), Hirvatistan (Grgasović, 1997), Adriyatik Karbonat Platformu (Dinaridler) (Velić, 2007), Birleşik Arap Emirlikleri (Maurer vd., 2007), Endonezya (Al-Shaibani vd., 1983; Martini vd., 1997, 2004) ve Japonya (Chablais vd., 2010) ile benzerlik göstermektedir. Chablais vd. (2011) gerçekleştirmiş oldukları çalışma ile Geç Triyas foraminiferlerinin Tetis ve Pantalasa içerisindeki paleobiyocoğrafik dağılımlarından yararlanarak her biri karakteristik foraminifer topluluğu içeren 6 farklı bölge tanımlanmış olup, Türkiye'nin sı̆̆ denizel karbonatların hakim olduğu Tetis çevresinde yer aldığını ve İtalya, Yunanistan, Kıbris ve batı Karpatlar ile benzer foraminifer topluluklarına sahip olduğunu öne sürmüştür. Belirgin faunal benzerlikler, Geç Triyas zamanında, foraminiferlerin paleobiyocoğrafik dağılımlarının anlaşılmasında ve belirlenmesinde önemli bir anahtardır. Bitlis masifine ait Üst Triyas karbonatları, involutinid benzeri yaygın oportunistik-firsatçı formlarla birlikte ve textularid formlar, algler ve mercanlar içerdiğinden Tetis provensini karakterize etmektedir. Literatür verileri ve bu çalışmada tanımlanan foraminifer topluluklarının paleobiyocoğrafik yayılımı ve karbonatların mikrofasiyes özellikleri dikkate alındığıında, Geç Triyas döneminde Bitlis Masifi'nin paleocoğrafik konumunun, Gondvana'nın kuzey kenarına yakın, Neotetis Okyanusu'nun açılımına eşlik eden sığ denizel platform olduğu söylenebilir. 


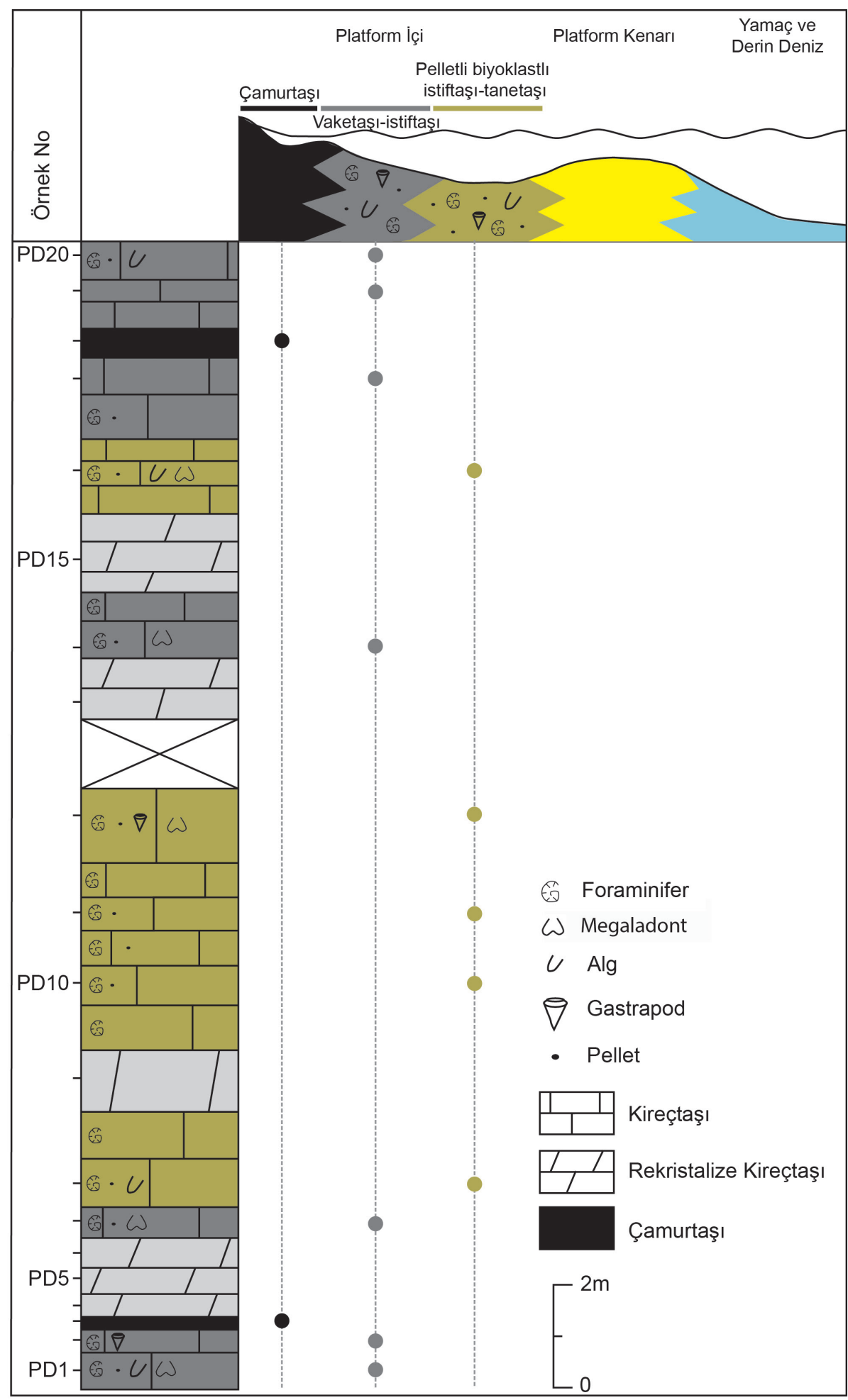

Şekil 7. Palu ölçülü kesiti boyunca tanımlanan mikrofasiyes tiplerinin dağılımları ve çökelim ortamları.

Figure 7. Distribution of defined microfacies types and their depositional environments in the Palu measured section. 


\section{SONUÇLAR}

Bitlis Masif'i içerisinde Palu (Elazığg) Çakmakkaya köyü civarında yüzeylenen Üst Triyas karbonatlı kayaçların biyostratigrafik ve mikrofasiyes özelliklerini ortaya koymak amacıyla gerçekleştirilen bu çalışmada, bentik foraminiferler kullanılarak biyostratigrafik çatı kurulmuştur. Çalışılan istifdeki mikropaleontolojik analizler bölgedeki Üst Triyas karbonatlarının platform ortam koşullarını karakterize eden foraminifer topluluklarını içerdiğini göstermektedir. Tetis provensine ait Üst Triyas sı̆̆ denizel sedimanların en önemli fosil gruplar1, Triasina, Aulotortus, Involutina cinslerini içeren involutinidlerdir. $\mathrm{Bu}$ formlar ölçülen kesitte tanımlanan ana foraminifer grubudur. $\mathrm{Bu}$ fosil grupları bir çok çalışmacı tarafından biyostratigrafik, bölgesel ve küresel ölçekte korelasyon amaçlı kullanılmıştır. İstif boyunca, Geç Noriyen-Resiyen aralığında k1lavuz fosil olarak kabul edilen Triasina hantkeni belirlenmiştir. Mikrofasiyes çalışmaları kapsamında, karbonat kayaçlarına ait dokusal, petrografik ve paleontolojik verilerin 1şı̆̆ında, birim içerisinde, sı ̆ denizel platform ve lagün ortamını karakterize eden üç temel mikrofasiyes tipi, çamurtaşı, vaketaş1-istiftaşı ve pelletli biyoklastlı istiftaşı-tanetaşı, tanımlanmıştır.

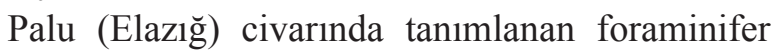
topluluğu ve fasiyes tipleri, Türkiye'de ve Dünya'da farklı bölgelerde gerçekleştirilen çalışmalara benzer foraminifer topluluklarına, fasiyes tiplerine ve çökelme ortamlarına sahip olduğu gözlenmiştir. $\mathrm{Bu}$ verilere dayanılarak, bölgenin, Geç Triyas döneminde Neotetis Okyanusu'nun güney kolunun aç1lımına bağlı olarak Gondvana kıtasından ayrılmış, sı ğ denizel platform ortamını karakterize ettiği sonucuna varılmıştır.

\section{KATKI BELIRTME}

$\mathrm{Bu}$ çalışma, TÜBİTAK 117Y485 no'lu proje kapsamında desteklenmiştir. Yazar, paleontolojik verilerin kontrol edilmesinde ve makalenin geliştirilmesinde yapıcı eleştirilerde bulunan Prof. Dr. Demir Altıner'e, makalenin son şeklini kazanmasında katkılar sağlayan Doç. Dr. Yavuz Özdemir'e ve makalenin değerlendirme sürecindeki değerli görüş ve önerileriyle katkı sağlayan Prof. Dr. Atike Nazik ve Prof. Dr. Sevinç Özkan-Altıner'e teşekkür eder.

\section{EXTENDED SUMMARY}

TThe results of a biostratigraphic and microfacies studies in the Upper Triassic shallow marine carbonates of Bitlis massif exposed in the vicinity of Çakmakkaya village of Palu (Elazı ğ, Turkey) are outlined in this study. The micropaleontological analysis emphasizes foraminiferal assemblages, dominated by the involutinids, lagenids, and textularids. In the measured section, the Triasina hantkeni biozone has been established based by the presence of Triasina hantkeni, an index species of the late Noriyen-Rhaetian interval. The stratigraphic range of this zone is defined by the range of the key species as late NorianRhaetian. In addition to eponymous species, Aulotortus sinuosus, Aulotortus friedli, Involutina sp., Galeanella sp., Duostomina sp., Glomospira sp., Glomospirella sp., Duotaxis birmanica, Siphovalvulina sp., and Reophax sp. are the other foraminiferal taxa recorded in the Upper Triassic carbonates. The Triasina hantkeni Zone has been recognized in Turkey (İşintek, 2002; Beccaletto vd., 2005; Ekmekçi vd., 2006; Okay ve Altıner, 2007).

The Palu section mainly consists of mud-supported and recrystallized limestones particularly rich in benthic foraminifera, megalodonts, gastropods, corals and algae. Based on the detailed microfacies analysis, the Upper Triassic carbonates of the section mainly consists of three distinct microfacies types corresponding to subtidal and intertidal depositional settings. Our interpretation of these facies is mainly based on field observations and 
thorough examination of sedimentological and paleontological characteristics of carbonates. Wackestone-packstone and pelloidal bioclastic packstone-grainstone are the characteristic facies types of subtidal deposits in the measured section. These facies types comprise relatively abundant involutinids and textularids and the other common fossils like gastropods and dasycladacean algae. They are interpreted to have been deposited in lagoonal, low-energy environments indicating open platform interior conditions due to the high abundance of shallow marine organisms within a micritic matrix. Recrystalization is commonly effective, and the limestones can be partially or totally recrystallized throughout the studied section. Unfossiliferous mudstone facies is characteristic of the intertidal deposits within a restricted lagoonal environment.

Biostratigraphic and microfacies studies of the Upper Triasic sediments highlighted a similar succession with Tethyan realm allowing precise biostratigraphic correlations. Paleoecological data obtained from biostratigraphic studies combined with facies characteristics suggests that the Upper Triassic sediments were deposited in a wide carbonate platform environment simultaneously the opening of the southern branch of Neotethys.

\section{ORCID}

Ayşe Atakul Özdemir (D https://orcid.org/0000-0003-0660-3139

\section{DEĞIINİLEN BELGELER}

Aktaş, G., and Robertson, A.H.F., 1990. Tectonic evolution of the Tethys suture zone in SE Turkey: evidence from the petrology and geochemistry of Late Cretaceous and Middle Eocene extrusives. In: Malpas, J. et Al. (Eds) Ophiolites, Oceanic Crustal Analogues. Proceedings of Symposium, Troodos 1987 Geological Survey Department, Cyprus, 311-328.
Aktaş, G., and Robertson, A.H.F., 1984. The Maden Complex, SE Turkey: evolution of a Neotethyan active margin. Geological Society, London, Special Publications, 17, 375-405.

Al-Shaibani, S.K., Altıner, D., Brönnimann, P., Carter, D.J., et Zaninetti, L., 1982. Triasina hantkeni Majzon, 1954 (Foraminifère), dans le Trias supérieur de la Téthys (Europe et Asie). Archive Des Sciences de Genève, 35, 137-142.

Al-Shaibani, S.K., Carter, D.J., and Zaninetti, L., 1983. Geological and micropalaeontological investigations in the Upper Triassic (Asinepe Limestone) of Seram, Outer Banda Arc, Indonesia. Archive Des Sciences de Genève, 36, 297-313.

Altıner, D., et Zaninetti, L., 1980. Le Trias dans la region de Pinarbasi, Taurus oriental, Turquie: unites lithologiques, micropaleontologie, milieux de. Rivista Italiana Di Paleontologia e Stratigrafia, 86, 705-760.

Beccaletto, L., Bartolini, A.-C., Martini, R., Hochuli, P.A., and Kozur, H., 2005. Biostratigraphic data from the Çetmi Melange, northwest Turkey: Palaeogeographic and tectonic implications. Palaeogeography, Palaeoclimatology, Palaeoecology, 221, 215-244.

Çağlayan, M.A., Önal, R.N., Şengün, M., and Yurtsever, A., 1984. Structural setting of the Bitlis Massif. Geology of the Taurus Belt. Proceedings of the International Symposium on the Geology of the Taurus Belt, Ankara, 245-254.

Chablais, J., Martini, R., Kobayashi, F., Stampfli, G., and Onoue, T., 2011. Upper Triassic foraminifers from Panthalassan carbonate buildups of Southwestern Japan and their paleobiogeographic implications. Micropaleontology, 57, 93-124.

Chablais, J., Onoue, T., and Martini, R., 2010. Upper Triassic reef-limestone blocks of southwestern Japan: New data from a Panthalassan seamount. Palaeogeography, Palaeoclimatology, Palaeoecology, 293, 206-222.

Ciarapica, G., et Zaninetti, L., 1984. Foraminifères et biostratigraphie dans le Trias supérieur de la série de la Spezia (Dolomies de Coregna et Formation de la Spezia, nouvelles forma- tions), Apennin septentrional. Revue de Paléobiologie, 117-134. 
Dunham, R.J., 1962. Classification of Carbonate Rocks according to Depositional Texture. American Association of Petroleum Geologists, 1, 108-121.

Ekmekçi, E., Özkan-Altıner, S., Altıner, D., Yılmaz, Ö., Erdoğan, K., Şener, S., Coşkun, B., Şenel, ve M., İşintek, İ., 2006. Torosların Geç Triyas-Liyas yaşlı istiflerinin foraminifer Biyostratigrafisi ve Mikrofasiyes özellikleri. MTA Rapor No. 10889, 73 s. (yayınlanmamış)

Flügel, E., 2004. Microfacies of Carbonate Rocks: Analysis, Interpretation and Application. SpringerVerlag Berlin Heidelberg. 976 p.

Gale, L., Kolar-Jurkovšek, T., Šmuc, A., and Rožič, B., 2012. Integrated Rhaetian foraminiferal and conodont biostratigraphy from the Slovenian Basin, eastern Southern Alps. Swiss Journal of Geosciences, 105, 435-462.

Gazdzicki, A., 1983. Foraminifers and biostratigraphy of Upper Triassic and Lower Jurassic of the Slovakian and Polish Carpathians. Acta Palaeontologica Polonica, 44, 109-169.

Gazdzicki, A., 1974. Rhaetian microfacies, stratigraphy and facial development in the Tatra Mis. Acta Geologica Polonica, 25, 17-120.

Göncüoğlu, M.C., and Turhan, N., 1984. Geology of the Bitlis Metamorphic Belt., Geology of the Taurus Belt. International symposium, Ankara, 237-245.

Grgasović, T., 1997. Upper Triassic biostratigraphy and algae from Žumberak (Croatia). Geologica Croatica, 50, 201-214.

Hall, R., 1976. Ophiolite emplacement and the evolution of the Taurus suture zone, southeastern Turkey. Bulletin of the Geological Society of America, 87, 1078-1088.

İşintek, İ., 2002. Foraminiferal and algal biostratigraphy and petrology of the Triassic to Early Cretaceous carbonate assemblages in the Karaburun Peninsula (Western Turkey). Tez, 263 s. (yayınlanmamış)

Kamoun, F., Martini, R., Peybernes, B., and Zaninetti, L., 1994. Micropalaeontological characterization of the "Rhaetian" along the North-South Axis (central Tunisia); comparison with the Rhaetian of the Dorsale and the Saharan Shelf $\mid$ Caracterisation micropaleontologique du 'Rhetien" dans l'axe Nord- Sud (Tunisie cent." Rivista Italiana Di Paleontologia e Stratigrafia, 100, 365-382.
Kamoun, F., Peybernès, B., Martini, R., Zaninetti, L., Vila, J.-M., Trigui, A., et Rigane, A., 1998. Associations de foraminifères benthiques dans les séquences de dépôt du Trias moyen?-supérieur de l'Atlas Tunisien central et méridional. Geobios, 31, 703-714.

Mackintosh, P.W., and Robertson, A.H.F., 2012. Late Devonian-Late Triassic sedimentary development of the central Taurides, S Turkey: Implications for the northern margin of Gondwana. Gondwana Research, 21, 1089-1114.

Mancinelli, A., Chiocchini, M., Chiocchini, R.A., and Romano, A., 2005. Biostratigraphy of Upper Triassic-Lower Jurassic carbonate platform sediments of the central-southern Apennines (Italy). Rivista Italiana Di Paleontologia e Stratigrafia, 111, 271-283.

Márquez, L., Calvet, F., Arnal, I., and Trifonova, E., 1994. Foraminiferal assemblage in the Isabena Formation, southern Pyrenees, Upper Triassic, Spain. Boletín de La Real Sociedad Española de Historia Natural, Sección Geológica, 89, 189-197.

Martini, R., Vachard, D., Zaninetti, L., Cirilli, S., Cornée, J.-J., Lathuilière, B., and Villeneuve, M., 1997. Sedimentology, stratigraphy, and micropalaeontology of the upper triassic reefal series in Eastern Sulawesi (Indonesia). Palaeogeography, Palaeoclimatology, Palaeoecology, 128, 157-174.

Martini, R., Zaninetti, L., Lathuillière, B., Cirilli, S., Cornée, J.J., and Villeneuve, M., 2004. Upper Triassic carbonate deposits of Seram (Indonesia): Palaeogeographic and geodynamic implications. Palaeogeography, Palaeoecology, 206, 75-102.

Maurer, F., Rettori, R., and Martini, R., 2007. Triassic stratigraphy, facies and evolution of the Arabian shelf in the northern United Arab Emirates. International Journal of Earth Sciences, 97, 765.

MTA. 2011. 1/100.000 ölçekli Elazığ K-43 paftası. Maden Tetkik ve Arama Genel Müdürlüğü, Ankara.

Okay, A.I., and Altıner, D., 2007. A condensed mesozoic succession North of İzmir: A fragment of the anatolide-tauride platform in the Bornova Flysch Zone. Turkish Journal of Earth Sciences, $16,257-279$. 
Okay, A.I., Arman, M.B., and Göncüoglu, M.C., 1985. Petrology and phase relations of the kyaniteeclogites from eastern Turkey. Contributions to Mineralogy and Petrology, 91, 196-204.

Okay, A.I., Zattin, M., and Cavazza, W., 2010. Apatite fission-track data for the Miocene Arabia-Eurasia collision. Geology, 38, 35-38.

Perinçek, D., 1990. Hakkari ili ve dolayının stratigrafisi, Güneydoğu Anadolu, Türkiye. Türkiye Petrol Jeologları Derneği Bülteni, 2, 21-68.

Perinçek, D., 1980. Bitlis metamorfitlerinde volkanitli Triyas. Türkiye Jeoloji Kurumu Bülteni, 23, 201211.

Robertson, A.H.F., 1998. Mesozoic-Tertiary tectonic evolution of the easternmost Mediterranean area: integration of marine and land evidence. Proceedings of the Ocean Drilling Program, 160 Scientific Results. 723-782.

Robertson, A.H.F., Parlak, O., Rızaoğlu, T., Ünlügenç, Ü., İnan, N., Tasli, K., and Ustaömer, T., 2007. Tectonic evolution of the South Tethyan ocean: evidence from the Eastern Taurus Mountains (Elaziğ region, SE Turkey). Geological Society, London, Special Publications, 272, 231-270.

Robertson, A.H.F., Parlak, O., Yıldırım, N., Dumitrica, P., and Tasl1, K., 2016. Late Triassic rifting and Jurassic-Cretaceous passive margin development of the Southern Neotethys: evidence from the Adiyaman area, SE Turkey. International Journal of Earth Sciences, 105, 167-201.

Schäfer, P., and Senowbari-Daryan, B., 1981. Facies Development and Paleoecologic Zonation of Four Upper Triassic Patch-Reefs, Northern Calcareous Alps Near Salzburg, Austria. In: Toomey, D.F. (Ed.), European Fossil Reef Models. SEPM Society for Sedimentary Geology, 241-259.
Şengün, M., 1993. Bitlis masifinin metamorfizması ve örtü çekirdek ilişkisi. Maden Tetkik ve Arama Dergisi, 115, 1-13.

Tunaboylu, B., Altıner, D., İşintek, I., and Demirci, D., 2014. Foraminiferal biostratigraphy and sequence stratigraphy of peritidal carbonates at the TriassicJurassic boundary (Karaburun Peninsula, Western Turkey). Journal of Asian Earth Sciences, 90, 61-76.

Varol, E., Tekin, U.K., and Temel, A., 2007. Age and geochemistry of middle to late carnian basalts from the alakirçay nappe (antalya nappes, sw turkey): Implications for the evolution of the southern branch of neotethys. Ofioliti, 32, 163-176.

Velić, I., 2007. Stratigraphy and Palaeobiogeography of Mesozoic Benthic Foraminifera of the Karst Dinarides (SE Europe). Geologia Croatica, 60, $1-86$.

Wilson, J.L., 1975. Carbonate Facies in Geologic History. Springer Verlag, New York.

Yılmaz, Y., Yiğitbaş, E., and Genç, C., 1993. Ophiolitic and metamorphic assemblages of southeast Anatolia and their significance in the geological evolution of the orogenic belt. Tectonics, 12, 1280-1297.

Yümün, Z.Ü., Kılıç, A.M., Martini, R., Metzger, J., and Tunç, M., 2013. Late Triassic and Lower Jurassic Foraminifera of the carbonate platform of the Beyaz Aladağ Group (Eastern Taurus, Turkey): New stratigraphic implications. Geobios, 46, 447459 .

Zaninetti, L., 1976. Les foraminiferes du Trias. Essai de synthese et correlation entre les domaines mesogeens europeen et asiatique. Rivista Italiana Di Paleontologia e Stratigrafia, 82, 1-258. 
